Draft version April 11, 2017

Preprint typeset using $\mathrm{LATE}_{\mathrm{E}} \mathrm{X}$ style emulateapj v. 6/22/04

\title{
NUSTAR HARD X-RAY OBSERVATIONS OF THE ENERGETIC MILLISECOND PULSARS PSR B1821-24, PSR B1937+21, AND PSR J0218+4232
}

\author{
E. V. GotThelF ${ }^{1}$ And S. Bogdanov \\ Columbia Astrophysics Laboratory, Columbia University, 550 West 120th Street, New York, NY 10027, USA \\ Draft version April 11, 2017
}

\begin{abstract}
We present Nuclear Spectroscopic Telescope Array (NuSTAR) hard X-ray timing and spectroscopy of the three exceptionally energetic rotation-powered millisecond pulsars PSRs B1821-24, B1937+21, and J0218+4232. By correcting the NuSTAR on-board clock for phase and frequency drifts between observation intervals, we are able to recover the intrinsic hard X-ray pulse profiles of all three pulsars with a resolution down to $\leq 15 \mu \mathrm{s}$. The substantial reduction of background emission relative to previous broad-band X-ray observations allows us to detect for the first time pulsed emission up to $\sim 50 \mathrm{keV}, \sim 20 \mathrm{keV}$, and $\sim 25 \mathrm{keV}$, for the three pulsars, respectively. We conduct phase-resolved spectroscopy in the $0.5-79 \mathrm{keV}$ range for all three objects, obtaining the best yet measurements of the broad-band spectral shape and high-energy pulsed emission to date. We find extensions of the same power-law continua seen at lower energies, with no evidence for a spectral turnover or break. Extrapolation of the X-ray power-law spectrum to higher energies reveals that a turnover in the 100 $\mathrm{keV}$ to $100 \mathrm{MeV}$ range is required to accommodate the high energy $\gamma$-ray emission observed with Fermi LAT, similar to the broad-band spectral energy distribution observed for the Crab pulsar.

Subject headings: pulsars: general — pulsars: individual (PSR B1821-24, PSR B1937+21, PSR J0218+4232) - stars: neutron - X-rays: stars
\end{abstract}

\section{INTRODUCTION}

Millisecond pulsars (MSPs) represent a distinct population of old rotation-powered pulsars, with short spin periods, $P \leq 25 \mathrm{~ms}$, and typically small intrinsic spindown rates, $\dot{P}_{i} \sim 10^{-20}$, implying relatively low surface magnetic dipole field strengths $B_{\text {surf }} \propto\left(P \dot{P}_{i}\right)^{1 / 2} \sim$ $10^{8}-10^{10} \mathrm{G}$ and large characteristic spindown ages $\tau \equiv P / 2 \dot{P}_{i} \geq 1$ Gyr. These objects are produced via spin-up by accretion of matter and angular momentum by a close stellar companion in a low-mass X-ray binary.

Of the $\sim 300$ known radio MSPs about dozens have detected in X-rays, and these can be broadly grouped into three distinct categories: (i) pulsed thermal radiation from the neutron star magnetic polar caps (e.g., Zavlin 2006; Bogdanov et al. 2006; Forestell et al. 2014); (ii) unpulsed shock emission due to a pulsar wind-driven intrabinary shock (e.g., Bogdanov et al. 2005, 2014); and (iii) pulsed non-thermal radiation from the pulsar magnetosphere (see, e.g., Zavlin 2007).

The MSPs in the latter category provides an important testbed for theoretical models of pulsar electrodynamics and emission physics because they exhibit remarkably similar properties to non-recycled young pulsars; this despite their faster spin (and as a result much lower altitude of the pulsar light cylinder) and weaker surface magnetic fields $\left(\sim 10^{8}-10^{9} \mathrm{G}\right)$. This is likely a consequence of their high magnetic fields in the vicinity of the light cylinder $\left(\sim 10^{5} \mathrm{G}\right)$, where the non-thermal radiation is thought to originate. Constraining the emission properties over as wide an photon energy range as possible may offer vital clues regarding the emission physics of MSPs, and by extension all pulsars. Specifically, important insight

1 Departament de Física Quàntica i Astrofísica, Institut de Ciències del Cosmos, Universitat de Barcelona, IEEC-UB, Martí i Franquès 1, 08028, Barcelona, Spain into the detailed physics of MSP magnetospheric emission can be gained by investigating the energy dependence of the pulsations and the broadband high-energy (X-rays through $\gamma$-rays) spectral properties of these pulsars.

In this paper we present the first $N u S T A R$ hard X-ray $(3-79 \mathrm{keV})$ observations of three magnetospheric driven MSPs, PSRs B1821-24 (J1824-2452A), B1937+21 $(\mathrm{J} 1939+2134)$, and $\mathrm{J} 0218+4232$, whose properties are given in Table 1. These are among the most energetic MSPs known and the only ones in their category sufficiently bright to measure above $10 \mathrm{keV}$. The NuSTAR data allows us to study their X-ray spectra in an energy range beyond what was previously possible, and to consider their broader spectral energy distribution (SED) with respect to Fermi spectra. To fully exploit the phase information for these rapidly spinning pulsars $(1.6-3.0 \mathrm{~ms})$ we devise a method to correct for the drift and fluctuation of the NUSTAR clock, whose short-term timing variance is otherwise on the order of the pulse periods for all three pulsars.

This work is laid out as follows. In $\S 2$, we present our NuSTAR observation PSR B1821-24, along with a spectroscopic reanalysis of archival $R X T E$ and Chandra data. Observations and data reduction of these data are detailed in $\S 2.1$. In $\S 2.2$ we explain our technique used to correct the photon arrival time for NuSTAR clock inaccuracies. The resulting phase-resolved spectral and image analysis for PSR B1821-24 are explained in $\S 2.3$ and the results presented in $\S 2.4$. In $\S 3$ we present a phaseresolved spectral analysis of the archival NuSTAR, Chan$d r a$, and XMM-Newton data for PSR B1937+21; the results are given in $\S 3.1$. Similarly, in $\S 4$ presents the observations and results of a spectroscopy analysis of PSR J0218+4232 using existing NuSTAR and XMM-Newton data. In $\S 5$ we discuss the SED for each pulsar and offer 
TABLE 1

Summary of Properties of MSPs Observed with NuSTAR

\begin{tabular}{|c|c|c|c|c|c|c|c|c|}
\hline $\begin{array}{c}\text { Pulsar }^{1} \\
\text { (PSR name) }\end{array}$ & $\begin{array}{c}\text { R.A. } \\
\text { (J2000) }\end{array}$ & $\begin{array}{c}\text { Decl. } \\
(\mathrm{J} 2000)\end{array}$ & $\begin{array}{c}d \\
(\mathrm{kpc})\end{array}$ & $\begin{array}{c}P \\
(\mathrm{~ms})\end{array}$ & $\dot{P}$ & $\begin{array}{c}\dot{E} \\
\left(\operatorname{erg~s}{ }^{-1}\right)\end{array}$ & $\begin{array}{c}B \\
(\mathrm{G})\end{array}$ & Type \\
\hline $\mathrm{J} 0218+4232$ & 021759.9 & +423223 & 3.15 & 2.323 & $7.74 \times 10^{-20}$ & $2.44 \times 10^{+35}$ & $4.29 \times 10^{+8}$ & Magnetospheric \\
\hline J0437-4232 & 043721.0 & $-47 \quad 1549$ & 0.16 & 5.757 & $5.73 \times 10^{-20}$ & $1.19 \times 10^{+34}$ & $5.81 \times 10^{+8}$ & Thermal \\
\hline $\mathrm{J} 1023+0038$ & 102336.5 & +003806 & 1.37 & 1.688 & $1.20 \times 10^{-20}$ & $9.85 \times 10^{+34}$ & $1.44 \times 10^{+8}$ & Intra-binary shock \\
\hline $\mathrm{J} 1227-4853$ & 122801.9 & -485551 & 1.80 & 1.686 & $1.11 \times 10^{-20}$ & $9.13 \times 10^{+34}$ & $1.38 \times 10^{+8}$ & Intra-binary shock \\
\hline J1723-2837 & 172314.8 & -283910 & 0.72 & 1.856 & $7.54 \times 10^{-21}$ & $4.66 \times 10^{+34}$ & $1.20 \times 10^{+8}$ & Intra-binary shock \\
\hline B1821-24 & 182434.6 & -244828 & 5.50 & 3.054 & $1.62 \times 10^{-18}$ & $2.24 \times 10^{+36}$ & $2.25 \times 10^{+9}$ & Magnetospheric \\
\hline B1937+21 & 193930.0 & +213401 & 3.50 & 1.558 & $1.05 \times 10^{-19}$ & $1.10 \times 10^{+36}$ & $4.09 \times 10^{+8}$ & Magnetospheric \\
\hline J2339-0533 & 233936.5 & -053404 & 1.10 & 2.884 & $1.41 \times 10^{-20}$ & $2.32 \times 10^{+34}$ & $2.04 \times 10^{+8}$ & Intra-binary shock \\
\hline
\end{tabular}

${ }^{a}$ The NuSTAR observations of the center of 47 Tuc, which contains at least 25 MSPs (Pan et al. 2016), are not included in this Table. X-ray emission from the MSPs in 47 Tuc is completely dominated by the bright overlapping LMXB X9 (Bahramian et al. 2017) and are not detectable.

conclusions in $\S 6$.

\section{PSR B1821-24}

The bright 3.05 ms pulsar PSR B1821-24 (also known as PSR J1824-2452A) in the globular cluster M28 has the distinction of being the most energetic MSP known, with a spin-down luminosity ${ }^{2}$ of $\dot{E}=2.2 \times 10^{36} \mathrm{erg} \mathrm{s}^{-1}$ and was the first one discovered in a globular cluster (Lyne et al. 1987). This pulsar is also the first MSP from which non-thermal pulsed X-rays were detected (using $A S C A$; Saito et al. 1997). Nearly all of the X-ray emission is contained in two exceptionally narrow pulses, with a duty cycle of only a few percent (Rutledge et al. 2004; Ray et al. 2008).

PSR B1821-24 was well observed in X-rays using the Chandra and RXTE observatories. Its non-thermal spectrum is characterized by an absorbed power-law model with photon index of $\Gamma \approx 1.3$ and luminosity of $L(0.3$ $8 \mathrm{keV} ; 5.5 \mathrm{kpc})=1.4 \times 10^{33} \Theta \mathrm{erg} \mathrm{s}^{-1}$, where $\Theta$ is an unknown beaming fraction of the X-ray emission pattern (Bogdanov et al. 2011; Rots et al. 1998). In this sense, PSR B1821-24 is more similar to young, energetic pulsars than a typical MSPs. RXTE measurements sensitive in the hard X-ray band $(2-100 \mathrm{keV})$ are only able to detected pulsations up to $\sim 15 \mathrm{keV}$ despite deep observations (see e.g., Rots et al. 1998; Ray et al. 2008). Whether this is an intrinsic cut-off on the pulsar flux or an instrumental limitation was yet unknown.

In the following subsections we present $N u S T A R$ observations of PSR B1821-24, analyzed together with the accumulated Chandra and $R X T E$ data, that allows us to extend the previous spectral results up to $79 \mathrm{keV}$.

\subsection{OBSERVATIONS AND ANALYSIS}

\subsubsection{NuSTAR}

NuSTAR observed the globular cluster M28 on 2015 June 6 (ObsId 30101053002) followed by a second observation, 3.6 days later (ObsId 30101053004). NuSTAR consists of two co-aligned X-ray telescopes, with corresponding focal plane modules FPMA and FPMB that provide $18^{\prime \prime}$ FWHM imaging resolution over a $3-79 \mathrm{keV}$

\footnotetext{
2 The measured spin-down rate $\dot{P}$ and derived spin-down luminosity $\dot{E} \propto \dot{P} / P^{3}$ is not significantly affected by acceleration of the pulsar in the globular cluster potential (see Johnson et al. 2013).
}

X-ray band, with a characteristic spectral resolution of $400 \mathrm{eV}$ FWHM at $10 \mathrm{keV}$ (Harrison et al. 2013). The reconstructed $N u S T A R$ coordinates are accurate to $7^{\prime \prime} .5$ at the $90 \%$ confidence level. The nominal timing accuracy of $N u S T A R$ is $\sim 2 \mathrm{~ms}$ rms, after correcting for drift of the on-board clock, with the absolute timescale shown to be better than $<3 \mathrm{~ms}$ (Mori et al. 2014; Madsen et al. 2015). Although this is insufficient to resolve the $3.05 \mathrm{~ms}$ period of PSR B1821-24, we devise a method, presented herein, to correct the photon arrival times to better than $15 \mu$ s accuracy.

The data were reprocessed and analyzed using FTOOLS 09May2016_V6.19 (NUSTARDAS 14Apr16_V1.6.0) with NuSTAR Calibration Database (CALDB) files of 2016 July 6 . The resulting data set provides a total of $140.7 \mathrm{ks}$ and $55 \mathrm{ks}$ of net good exposure time for the two pointings, respectively. For the timing analysis, the nominal $N u S T A R$ clock correction was applied to the photon arrival times that are converted to barycentric dynamical time (TDB) using the radio timing coordinates and the DE405 solar system ephemeris. To search for the pulsar signal we extracted photon arrival times from each detector/observation pair using a $r=0.5^{\prime}$ circular aperture centered on the detected pulsar location and merged data from both FPM detectors.

Although the globular cluster M28 hosts at least 11 other pulsars (Bégin 2006), they are all over a factor of $\sim 100$ fainter than PSR B1821-24 in X-rays (Bogdanov et al. 2011) and thus cannot be studied effectively with $N u S T A R$.

\subsection{2. $R X T E$}

PSR B1821-24 has been observed extensively in hard X-rays with $R X T E$ during the mission lifetime, primarily for the purpose of absolute spacecraft time calibration and to measure the X-ray-to-radio phase lags (Rots et al. 1998; Rav et al. 2008). Here we collectively analyze all available archived $R X T E$ data on PSR B1821-24 for the first time. A total of $180 \mathrm{ks}$ of exposure time was accumulated during three programs: P20159 (1997 Feb 12-13, $16.9 \mathrm{ks}$ ), P90053 (2005 Feb 28-Mar 1, 14.9 ks), P92008 (2006 Jul 6-8,48.4 ks ; 2006 Oct 9-10,51.2 ks ; 2007 Apr $25-26,48.5 \mathrm{ks})$. These observations were made with the Proportional Counter Array (PCA; Jahoda et al. 1996) on-board RXTE. The PCA consists of five collimated xenon proportional counter units (PCUs), each having 
a front propane anticoincidence layer, with a total effective area of $\sim 6500 \mathrm{~cm}^{-2}$ over a $1^{\circ}$ field of view (FWHM). Each PCU is sensitive to photons in the energy range of $2-60 \mathrm{keV}$, with an energy resolution of $18 \%$ at $6 \mathrm{keV}$ recorded in 256 channels.

Data were collected in GoodXenonwithPropane mode with 2-5 PCU modules in use at any given time, with an average of 3.7 PCUs active overall. In this mode, photon arrival times are recorded with $1 \mu s$ resolution. We analyzed events from the top xenon layers of each PCU only. Including the second layers resulted in no significant improvement in the signal-to-noise ratio. The data were time filtered using standard criteria and analysis using the RXTE FTOOLS package. The arrival times for all data were corrected to the solar system barycenter using the JPL DE200 planetary ephemeris and the radio coordinates.

$R X T E$ PCA phase-resolved light curves and spectra for PSR B1821-24 are constructed using fasebin and related FTOOLs. By folding on the radio ephemeris of Ray et al. (2008; see their Table 1) we obtained a summed spectrum in 100 phase bins including data from the 22 observation segments. Energy selected light curves were generated using fbssum and a combined spectrum comprising data from all RXTE programs are added using fbadd. Appropriate response matrix were created for each PCU using pcarsp for the phased average spectra extracted using seextrct.

\subsubsection{Chandra}

In our analysis, we also make use of archival Chandra ACIS-S imaging and spectroscopic data of M28 from 2002 (ObsIDs 2684, 2685, 2683) and 2008 (ObsIds 9132,9133$)$ totaling $240 \mathrm{ks}$ and previously presented in Becker et al. (2003), Bogdanov et al. (2011), and Servillat et al. (2012). We also consider Chandra HRC-S observations of PSR B1821-24 obtained in SI mode (ObsIDs 2797 and 6769), which offer a $16 \mu$ s time resolution but no useful spectral information. The data reduction and analysis procedures of all Chandra observations are fully detailed in Bogdanov et al. (2011).

\subsection{Correcting the NuSTAR Photon Arrival Times}

Before we can take advantage of the $\mu$ s time resolution available with the NUSTAR FPM imagers we must address several clock issues relevant to observing MSPs. The NUSTAR clock frequency is known to drift at a steady rate over time and is typically adjusted every few days by means of a coarse divisor to limit the wandering from a nominal $240 \mathrm{MHz}$ rate. A model of the clock drift is stored in the clock correction file and used by the FTOOL barycorr to correct the NuSTAR photon arrival times in conjunction with the barycenter correction. However, unaccounted for rms residuals of order $\sim 2$ ms remain in the clock drift model.

In addition, spacecraft times are adjusted to the Coordinated Universal Time system during telemetry downlinks, several times a day. This can introduce a phase shift due to an uncalibrated $\sim 1 \mathrm{~ms}$ relative offset in the absolute timestamps between ground stations. To mitigate the latter potential problem, all data for the PSR B1821-24 observations were acquired using a single (Malindi) ground station, as was used for the other two pulsars discussed herein.
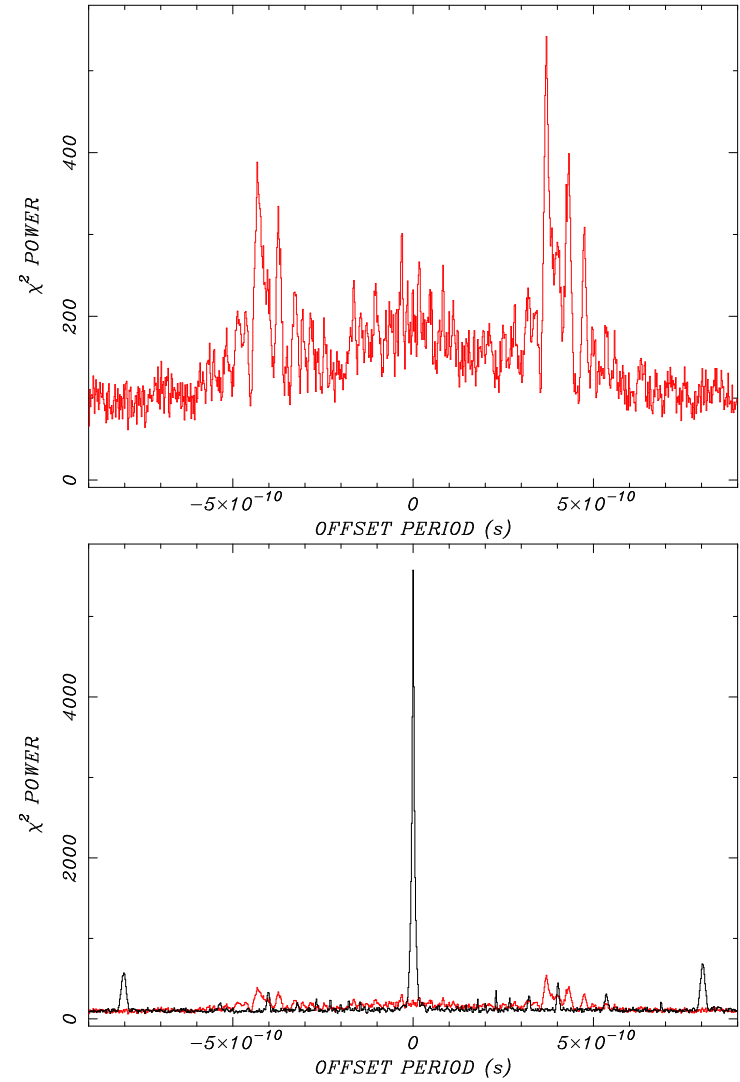

FIG. 1.- Periodogram of the PSR B1821-24 NuSTAR FPMA+FPMB data folded around the expected $3.05 \mathrm{~ms}$ spin period. Top: result using data processed using the standard methods available. Power in the pulsed signal is spread out over a number of "sidebands" around the expected period. Bottom: result for the same data after correcting for telescope clock inaccuracies using the method described in the text (black). The periodogram of the uncorrected data (red) from the upper panel is reproduced for reference.

Most consequential for fast pulsar timing is the variability of the clock rate on orbital timescales $(97 \mathrm{~min}$ ). Our investigation of the NuSTAR timing data reveals that the clock is stable to better than at least $15 \mu s$ during the spacecraft night, when the Solar illumination of the spacecraft is Earth blocked. However, during spacecraft day, the clock evidently wanders sufficiently between orbit-to-orbit observing intervals to lose phase cycle count for the $3 \mathrm{~ms}$ pulsar. Thus it is not possible to recover a coherently pulsar signal over several orbits. This is best illustrated by a pulsar search for PSR B1821-24 around the expected frequency using the nominal $\mathrm{NuS}$ $T A R$ data. As shown in the top panel of Figure 1 the search periodogram yields a complex power spectrum of low significance with a multitude of "sidebands" spanning $\delta f \approx 9 \times 10^{-5} \mathrm{~Hz}$.

Fortunately, the observations of PSR B1821-24 were taken exclusively during intervals of Earth block and its pulsed signal is sufficiently strong to measure a pulse frequency and phase over a single orbit. For the 60 orbits that comprise the full data set, on average 75 photons per orbit were collected in the source aperture for a typical $3.3 \mathrm{ks}$ of exposure. Detecting a signal with such few photons is uniquely possible because of the very sharp, asymmetric double peak profile, with $\approx 100 \%$ modula- 


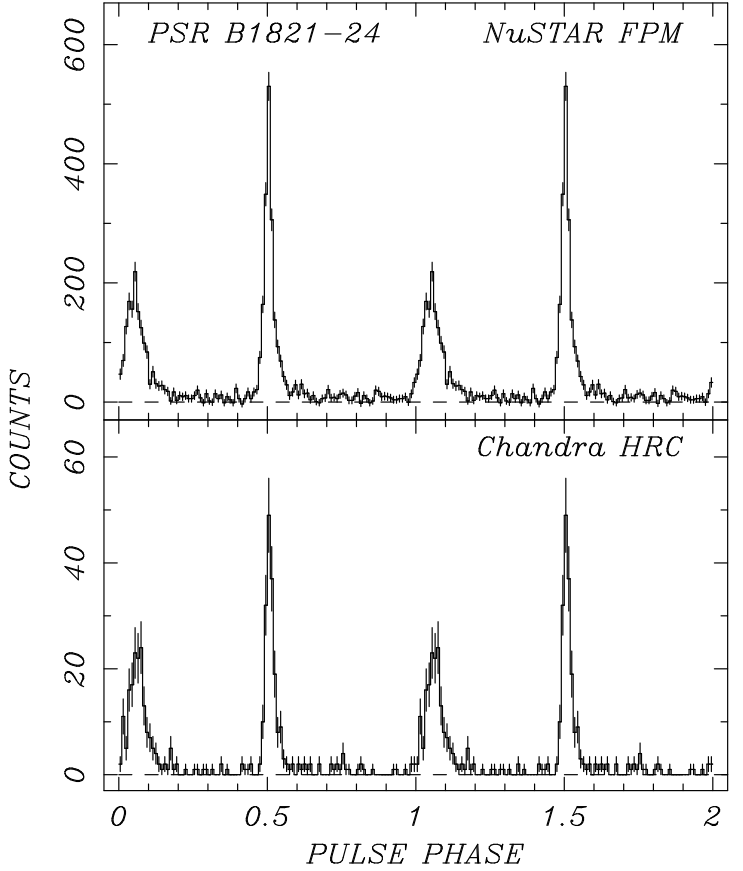

FIG. 2.- $3.05 \mathrm{~ms}$ pulse profiles of PSR B1821-24 using (Top) NuSTAR FPMA+FPMB 3-79 keV photon arrival times corrected for spacecraft clock inaccuracies, describe in this work, and (Bottom) Chandra HRC data obtained in timing mode. The two data sets complement each other, with HRC data dominant at energies below $2 \mathrm{keV}$ and NuSTAR above $3 \mathrm{keV}$. The background is not subtracted. The profile contains 100 phase bins and phase zero is arbitrarily aligned so that the pulsar peak $\mathrm{P} 1$ falls on $\phi=0.5$. Two cycles are shown for clarity.

tion and very low detector background. We use this fact to correct for frequency and phase errors introduced between orbits.

Our method is to break up the observation into orbitsized segments and correct for the advanced or retarded clock rate by adjusting the photon arrival times in the following sense,

$$
t^{\prime}=t \times \alpha_{i}+\beta_{i}
$$

where, for the $i^{\text {th }}$ segment, $\alpha_{i}$ is the ratio of the observed frequency of the pulsar relative to the expected frequency and $\beta_{i}$ is the phase offset at the adjusted times relative to the expected phase. The scale factors $\alpha$, around $\pm 4 \times 10^{-8}$, are measured using the $\chi^{2}$ statistic, optimal for the complex pulse shape. The phase offsets $\beta$ are determined by cross-correlating the pulse profile for each segment with an iterated high-statistic template. The $\beta$ 's insure that the scaled photon arrival times for each orbit align on the fiducial ephemeris relative to start of the observation, with the primary peak (P1) adjusted to phase $\phi_{P 1}=0.5$. This method accounts for all unmodeled orbit-to-orbit clock shifts.

To constructed the set of $60 \alpha$ 's and $\beta$ 's for PSR B1821-24, we extracted photons from an optimal combination of source aperture $\left(r=0.85^{\prime}\right)$ and energy range $(3-25 \mathrm{keV})$ that maximizes the pulsar signal and cross-correlation the folded light curves for each segment in 100 phase bins with the template. We applied these coefficients to the photon arrival times to generate the corrected event files that can now be coherently folded on the input ephemeris to recover the full pulsar signal ${ }^{3}$ and are used in all subsequent phase-resolved imaging and spectral analysis presented below. The resulting periodgram search using the corrected event times is shown in the bottom panel of Figure 1.

\subsection{NuSTAR Timing Analysis}

The folded light curve, using data merged from both NuSTAR pointings of PSR B1821-24, is able to fully resolve the peak of the pulsed signal in $30 \mu$ s phase bins. In Figure 2] we show the $3-20 \mathrm{keV} \mathrm{NuSTAR}$ pulse profile, alongside the Chandra HRC pulse profile, most sensitive below $2 \mathrm{keV}$. The morphology of the two profiles are very similar, showing the same sharp, asymmetric peak, despite the non-overlapping energy bands; there is no clear energy dependence.

Figure 3 also compares the NuSTAR and RXTE pulse profiles in two energy bands, $3-15$ and $15-79 \mathrm{keV}$. In the lower energy band, the profiles from the two missions are again strikingly similar. However, above $15 \mathrm{keV}$ the signal in the $R X T E$ data is barely visible above the background, while the $N u S T A R$ pulsations are clearly detected with high signal to noise ratio, up to $\sim 30 \mathrm{keV}$. This pulse signal is evidently not cutoff below $15 \mathrm{keV}$, but it is instead lost in the non-imaging $R X T E$ background. These light curves illustrates that the timing accuracy of the corrected $N u S T A R$ data set is on par with the highest quality RXTE and Chandra data.

\subsection{NuSTAR Image Analysis}

PSR B1821-24 is situated in the core of M28 where numerous other X-ray sources are present, as seen in the sub-arcsecond angular resolution Chandra images (Becker et al. 2003; Bogdanov et al. 2011). To NuSTAR imaging, the M28 cluster core is unresolved. This does not pose a problem since PSR B1821-24 is the brightest source above $\sim 5 \mathrm{keV}$. Indeed, a bright hard X-ray source is clearly detected at a position fully consistent with that of PSR B1821-24. The transient IGR J1824-24525 in M28 occasionally reaches comparable or higher luminosities and exhibits a hard spectrum (Papitto et al. 2013; Linares et al. 2014). However, this object spends the vast majority of the time in a dormant state, with luminosity $\sim 10^{31} \mathrm{erg} \mathrm{s}^{-1}$ so its quiescent emission in the hard X-ray band during the NuSTAR exposure is negligible for all practical purposes.

Figure 4 presents 3-20 keV exposure-corrected images of the NuSTAR M28 field smoothed using a $\sigma=3 !^{\prime \prime} 7$ Gaussian kernel and scaled linearly. To look for possible unpulsed X-ray emission from PSR B1821-24 we examined the NuSTAR phase-average, on-pulse and off-pulse images. We define the phase interval corresponding to the on-pulse so as to include both the P1 peak (10/100 phase bins) and the P2 peaks (16/100 phase bins; see Figure 2. The off-pulse interval contains the remaining phase bins.

The phase-averaged $N u S T A R$ image shows the bright pulsar, as well as emission coincident with Chandra source \#4 reported by Becker et al. (2003), likely an active galactic nucleus or a cataclysmic variable, to the

3 However, we can not be sure that phase count was strictly maintained. For example, if phase count was lost, it may not be possible to fold coherently a second pulsar in the field using the corrections for PSR B1821-24. 

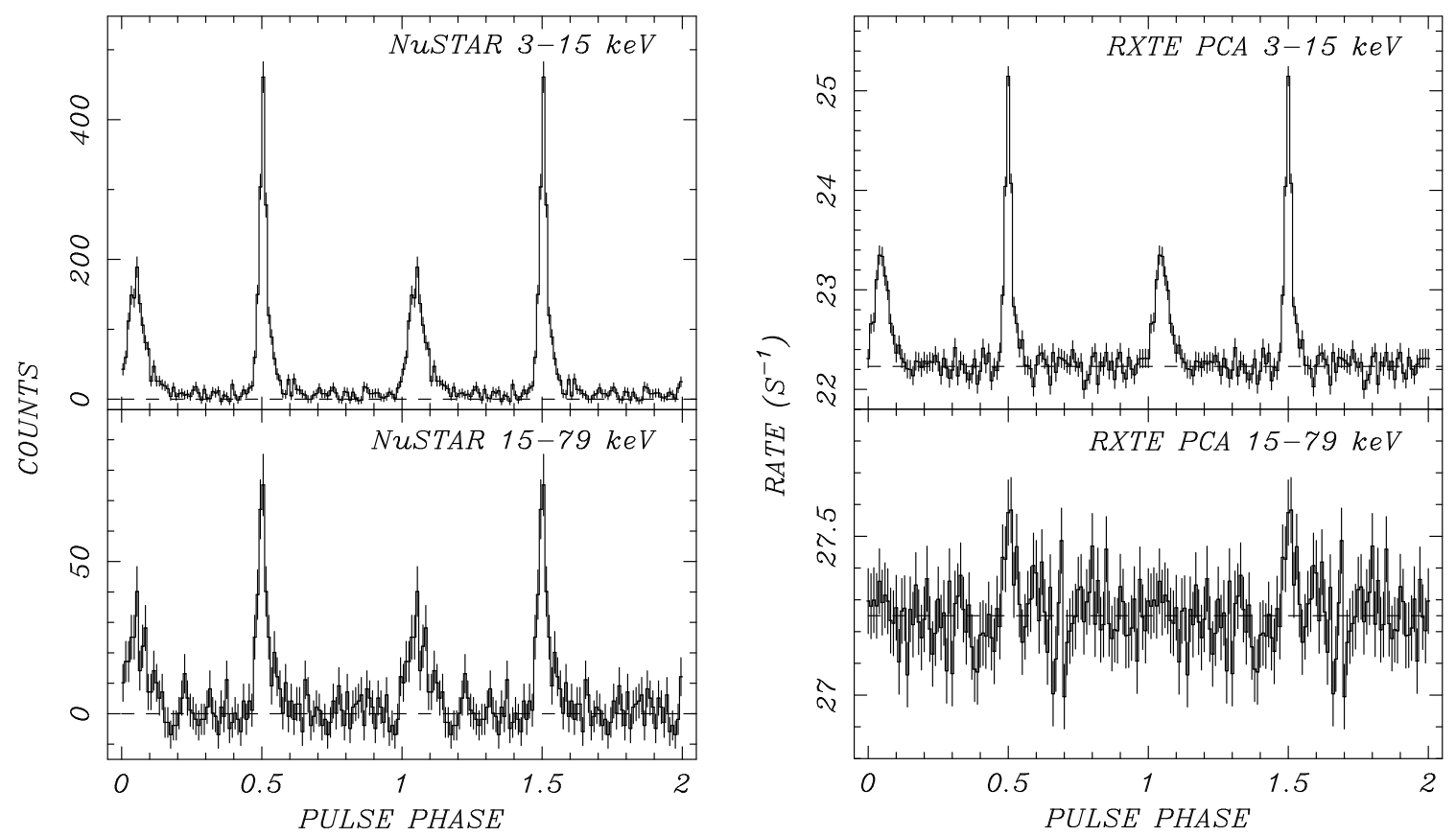

FIG. 3.- Comparison of the NuSTAR (Left) and RXTE (Right) 3.05 ms pulse profiles of PSR B1821-24 in M28 in two energy bands. The NuSTAR photon arrival times have been corrected for spacecraft clock inaccuracies, as described in the text. The background is not subtracted. These profiles contains 100 phase bins and phase zero is arbitrarily aligned so that the pulsar peak P1 falls on $\phi=0.5$. Two rotational cycles are shown for clarity.

northwest of PSR B1821-24. Also evident in the image is a large bright crescent of emission that corresponds to contamination from single bounce photons off the $\mathrm{NuS}$ $T A R$ optics originating from the bright X-ray binary GS 1826-24 (Ubertini et al. 1999), which is situated $1.6^{\circ}$ from the core of M28. From the off-pulse image it is clear that there is detectable emission at the location of the pulsar after accounting for the off-source background. The remainder can be attributed to hard X-ray emission from the numerous other sources in M28 that are unresolved by NuSTAR. We estimate this component from the scaled Chandra flux in the $3-10 \mathrm{keV}$ band that fall within the NuSTAR PSF. Also evident in the off-pulse image is an object just to the southwest of the pulsar consistent with the position of Chandra source \#17 identified by Becker et al. (2003), which is a candidate cataclysmic variable. The final panel of Figure 4 presents the off-pulse subtracted phase-average image, providing a clean representation of the NUSTAR PSF of the pulsar, as expected.

\subsection{X-ray Spectroscopy}

In all following spectral analyses, extracted spectra, grouped into appropriate channels, were fitted to an absorbed power-law spectrum model using the XSPEC (v12.8.2) package (Arnaud 1996). All spectral fits use the TBabs absorption model in XSPEC with the wilm Solar abundances (Wilms et al. 2000) and the vern photoionization cross-section (Verner et al. 1996).

\subsubsection{RXTE and Chandra Spectroscopy}

To isolate the purely pulsed emission for the $R X T E$ data, we used the scaled off-pulse phase intervals for the background spectrum. This provides a perfect representation of the non-pulsed emission. The resulting background-subtracted RXTE spectrum of PSR B1821-24 is then fit simultaneously with the $240 \mathrm{ks}$ Chandra ACIS-S3 (spatially isolated but phase-averaged) pulsar spectrum presented in Bogdanov et al. (2011), with their normalizations unlinked.

The available $R X T E$ data contains no useful spectral information above $\sim 15 \mathrm{keV}$ This is not necessarily due to the background, which exceeds the source rate by an order-of-magnetude at all energies, but rather for the lack of source photons and the large uncertainty in subtracting two comparably large numbers. With the column density fixed to the Chandra value, the best fit $\left(\chi^{2}=0.88\right.$ for 198 d.o.f.) power-law model in the $<15 \mathrm{keV}$ band yields a photon index of $\Gamma=1.25_{-0.14}^{+0.12}$, in agreement with Chandra result at lower energies. There is no evidence for flux variability. This result represents the best X-ray spectroscopy available for PSR B1821-24 prior to the NUSTAR result presented below.

\subsubsection{NuSTAR Spectroscopy}

We can improve on the $R X T E$ result by taking advantage of the greatly decreased NuSTAR background to extend the spectra measurements up to $79 \mathrm{keV}$. As for the $R X T E$ phase-resolved spectroscopy analysis, we isolate the NuSTAR spectrum of the pulsar into two pulse peaks and define a clean representation of the background using the scaled off-pulsed spectrum. Spectra were produced using the nuproducts script in the NuSTAR FTOOL package acting on the phase-resolved data files. The spectra are fitted using instrument and mirror response files generated for the phase-average spectra. We add the spectra derived from the two detectors and two observations to produce our final phase-averaged, on-pulse and off-pulse spectral and weighted response files.

We fitted the Chandra and NuSTAR spectra simulta- 

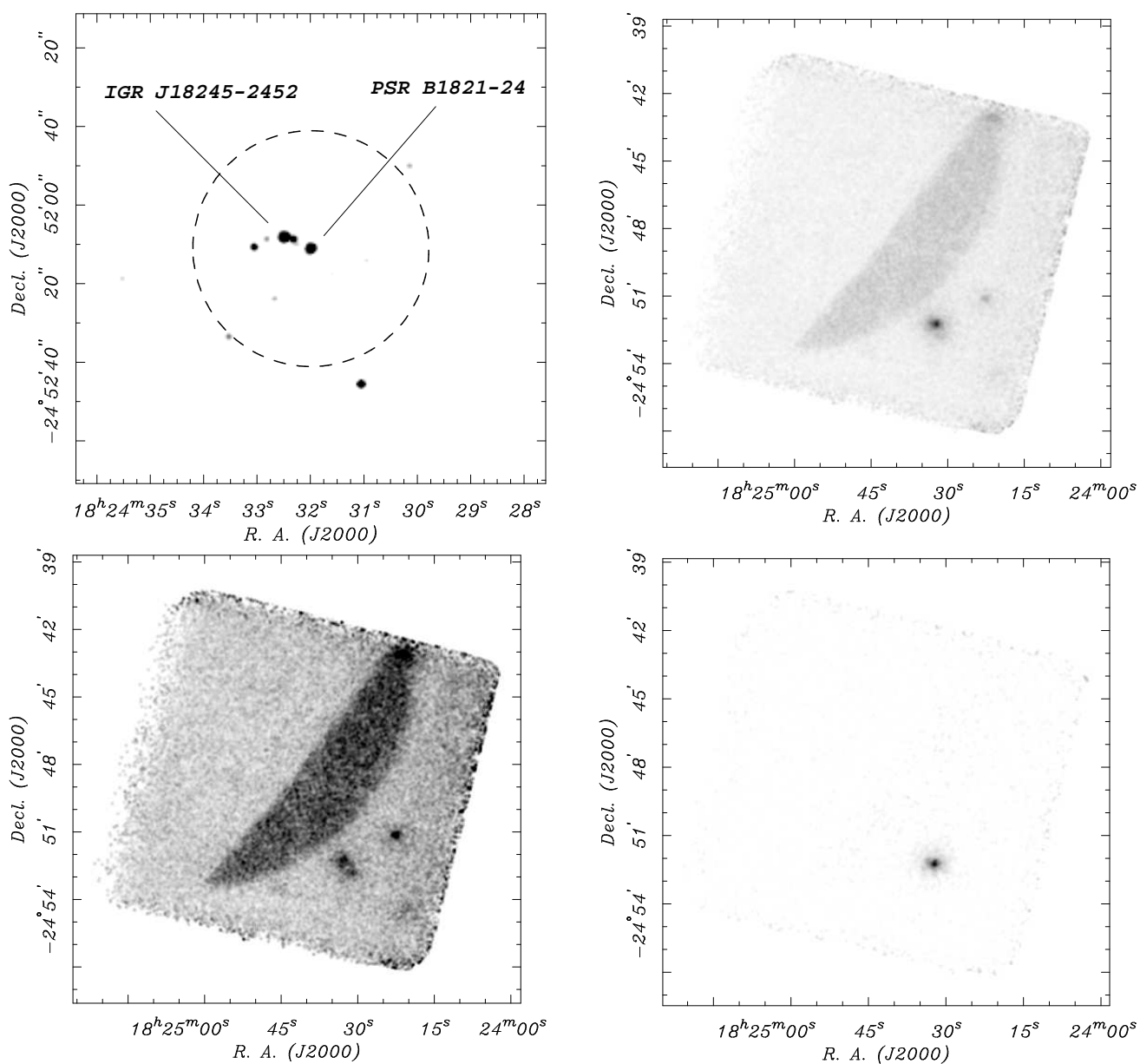

Fig. 4.- Top left - A reference high-resolution Chandra ACIS-S 3-10 kev image of the core of M28. The dashed circle of radius 0.5' centered on PSR B1821-24 corresponds to the source extraction region used for the NuSTAR analysis. In this observation from 2008, the transient source IGR J18245-2452 (Papitto et al. 2013) was more luminous than PSR B1821-24 but was $\sim 2$ orders of magnitude fainter at the time of the NuSTAR observations in 2015. PSR B1821-24 is by far the brightest persistent source in M28 above $\sim 3 \mathrm{keV}$. Other three panels - NuSTAR exposure-corrected and smoothed 3-20 keV X-ray images of the field containing PSR B1821-24. Top right The pulse phase averaged image showing the pulsar and source \#4 from Becker et al. (2003). Also evident is stray light contamination from the luminous X-ray binary GS 1826-24. Bottom left - The off-pulsar image that shows evidence of contamination from unresolved coincident hard sources in M28. Bottom right - The pulsar image generated by subtracting the off-pulse image from the phase-average image.

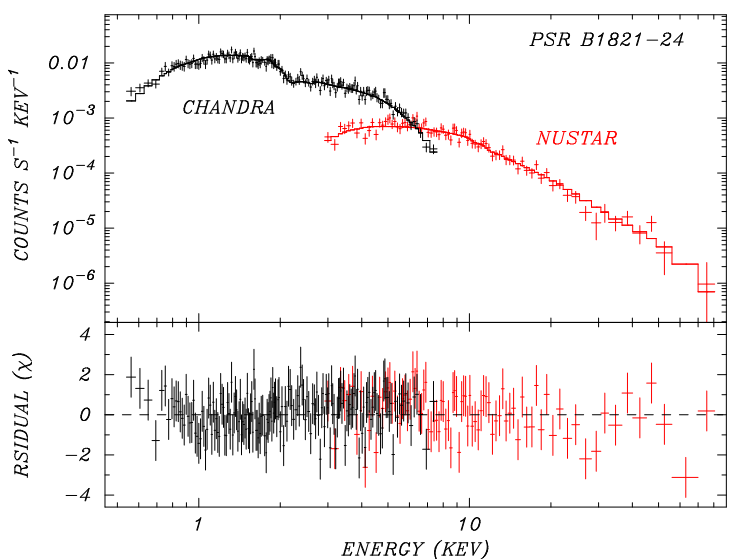

FIG. 5.- The NuSTAR FPMA+FPMB phase-resolved and Chandra ACIS-S phase-averaged spectra of PSR B1821-24. The two data sets are fitted simultaneously to an absorbed power-law model with their normalizations left to vary independently. The upper panel present NuSTAR (black) and Chandra (red) spectral histograms along with the best-fit model (solid lines) given in the text and Table 2 The lower panel shows the residuals from the best-fit model in units of sigma. neously to an absorbed power-law model over the entire $0.5-79 \mathrm{keV}$ energy band (see Figure 5). The resulting best fit spectral photon index is $\Gamma=1.28 \pm 0.05$ with $\chi_{\nu}^{2}=0.91$ for 226 degrees of freedom. The absorbed NuSTAR flux in the $2-10 \mathrm{keV}$ band is $(3.7 \pm 0.2) \times$ $10^{-13} \mathrm{erg} \mathrm{cm}^{-2} \mathrm{~s}^{-1}$ and $(3.8 \pm 0.1) \times 10^{-13} \mathrm{erg} \mathrm{cm}^{-2} \mathrm{~s}^{-1}$ for the Chandra spectrum. All quoted uncertainties are at a $90 \%$ confidence level. For a distance to M28 of $5.5 \mathrm{kpc}$ (Servillat et al. 2012), the derived fluxes imply an X-ray luminosity of $4 \times 10^{33} \Theta \mathrm{erg} \mathrm{s}^{-1}$ in the $0.3-79 \mathrm{keV}$ range. There is no requirement for an additional thermal component such as may arise due to heated polar caps. We also examined spectra from each pulse individually and find not significent difference in the photon index. The unabsorbed flux ratio of the two pulses is $P 1 / P 2=1.26$. A summary of the spectral results is given in Table 2 There are likely pile-up distortions of the Chandra spectrum at a level of a few percent that are difficult to accurately model. Nevertheless, as noted by Bogdanov et al. (2011), the effect on the measured parameters is negligible. 


\section{PSR B1937+21}

PSR B1937+21 (also referred to as PSR J1939+2134) was the first MSP discovered (Backer et al. 1982) and, for 25 years, the fastest spinning pulsar known $(P=$ $1.55 \mathrm{~ms})$. It is an isolated pulsar in the field of the Galaxy at a distance of $\sim 3.5 \mathrm{kpc}$. The spin-down luminosity of $\dot{E}=1.1 \times 10^{36} \mathrm{erg} \mathrm{s}^{-1}$ is the second highest among the MSP population. At X-ray energies, PSR B1937+21 has been detected as a pulsed source with ASCA (Takahashi et al.]2001), BeppoSAX (Nicastro et al. 2004), RXTE (Guillemot et al. 2012), Chandra and XMM-Newton (Ng et al. 2014). In hard $\mathrm{X}$-rays, the pulsar has so far only been detected up to $\approx 13 \mathrm{keV}$ with RXTE (Guillemot et al. 2012). Like PSR B1821-24, this MSP shows sharp pulsations with a small duty cycle and nearly $100 \%$ pulsed fraction.

\subsection{Observations and Results}

We examined the $41 \mathrm{ks}$ NuSTAR observation of PSR B1937+21 obtained on 2015 Aug 29 as part of the Cycle 1 Guest Observer program (ObsID 30101031002; PI Ng). These data were extracted, reduced, and analyzed in the same fashion as described above for PSR B1821-24. For the timing correction, an average of 66 counts per orbit were collected during 14 segments. The pulse signal from PSR B1937+21 detected in a segment is sufficiently strong to allow us to reproduce the method presented in 2.2 to correct the photon arrival times. We used the most current published pulsar spin parameters from Desvignes et al. (2016). This allowed us to accurately phase align the pulse profile over the course of the entire NuSTAR observation.

PSR B1937+21 was also observed with XMM-Newton on 2010 March 29 for a total exposure of 66.9 ks (ObsID 0605370101). These data were originally presented in Ng et al. (2014). The EPIC pn data for this observation were collected in timing mode, which offers $30 \mu \mathrm{s}$ time resolution but imaging along one dimension only. This data is not used here for spectroscopy due to known spectral issues for this mode.

The folded light curves from the two missions are shown in Figure 6. It is clear that the NuSTAR pulse profile is fully resolved and is comparable to the best $X M M$-Newton timing mode observations, also shown in Ng et al. (2014). The hard X-ray pulse profile for PSR B1937+21 is qualitatively similar to that of PSR B1821-24, but with a much suppressed secondary peak. The profile of the main peak displays the same characteristic asymmetry clearly evident in PSR B1821-24 and is detectable up to $20 \mathrm{keV}$ with $\mathrm{NuS}$ $T A R$, while the faint secondary pulse is lost to the background above $8 \mathrm{keV}$.

For spectroscopic analysis of PSR B1937+21, we combined NuSTAR, XMM-Newton and Chandra data. Following the receipe described previously, we extracted a NuSTAR on-pulse spectrum of PSR B1937+21 and the off-pulse spectrum to use as background. The $X M M-N e w t o n$ spectrum we extracted from the EPIC MOS1/2 data following standard procedures as outlined in $\mathrm{Ng}$ et al. (2014). We also retrieved an archival $49.5 \mathrm{ks}$ Chandra ACIS-S observation obtained on 2005 Jun 26 (ObsID 5516). The latter data were processed and reduced as detailed in $\mathrm{Ng}$ et al. (2014). We used phase av-

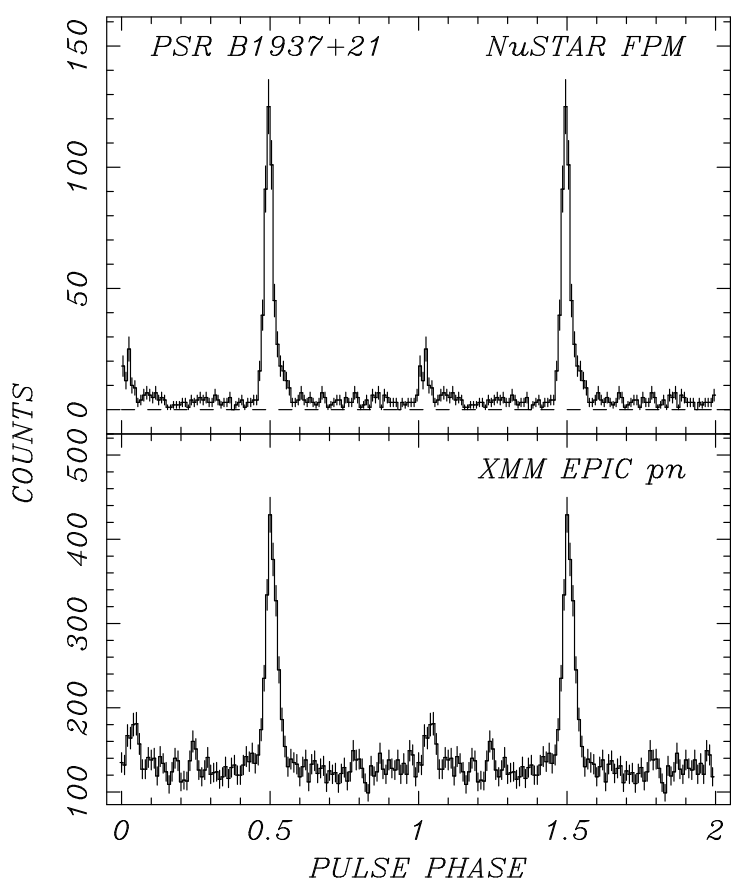

FIG. 6. $-1.55 \mathrm{~ms}$ pulse profiles of PSR B1937+21 using (Top) NuSTAR FPMA+FPMB 3-79 keV photon arrival times corrected for spacecraft clock inaccuracies, describe in this work, and (Bottom) XMM-Newton EPIC pn data obtained in fast timing mode. The background is not subtracted. These profiles contains 100 phase bins for NuSTAR and 60 for XMM-Newton and phase zero is arbitrarily aligned so that the pulsar peak P1 falls on $\phi=0.5$. Two cycles are shown for clarity.

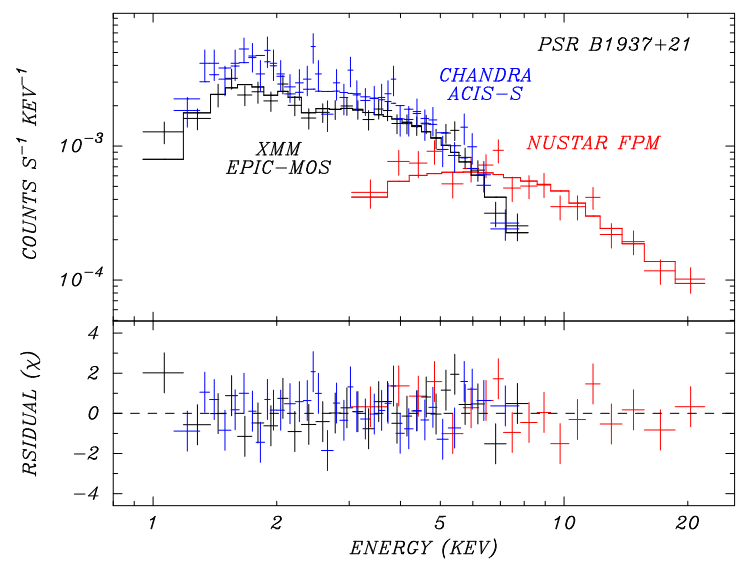

FIG. 7.- The NuSTAR FPMA+FPMB (red), Chandra ACIS-S (blue), and XMM-Newton EPIC MOS1+MOS2 (black) spectra of PSR B1937+21. The three spectra are fitted simultaneously to an absorbed power-law model with their normalizations left to vary independently. The upper panel present spectral histograms along with the best-fit model (solid lines) given in the text. The lower panel shows the residuals from the best-fit model in units of sigma.

eraged XMM-Newton and Chandra spectra as the time resolution of these data sets do not allow a phase-resolved spectral analysis.

The resulting simultaneously fit to the three data sets over the $0.5-25 \mathrm{keV}$ range is shown in Figure 7. Above $25 \mathrm{keV}$, the background flux dominates the NuSTAR spectrum. The best fit absorbed power-law model yields an absorbing column $N_{\mathrm{H}}=(1.78 \pm 0.27) \times 10^{22} \mathrm{~cm}^{-2}$ and spectral index $\Gamma=1.16 \pm 0.11$ with $\chi_{\nu}^{2}=0.91$ 
for 83 DoF. The measured NuSTAR 2-10 keV unabsorbed flux is $(2.37 \pm 0.29) \times 10^{-13} \mathrm{erg} \mathrm{s}^{-1} \mathrm{~cm}^{-2}$. The $X M M$-Newton flux is $10 \%$ higher, but consistent with imperfect background subtraction and local contamination (see $\mathrm{Ng}$ et al. 2014). The Chandra unabsorbed flux, $2.95 \pm 0.26 \times 10^{-13} \mathrm{erg} \mathrm{s}^{-1} \mathrm{~cm}^{-2}$, is also higher, by $20 \%$ at $1 \mathrm{keV}$ compared to the NuSTAR value, a difference significant at the $1.7 \sigma$ level. However, as for PSR B1821-24, there are likely pile-up distortions of the Chandra spectrum that artificially harden the intrinsic spectrum.

\section{PSR J0218+4232}

PSR J0218+4232 is a radio luminous $2.32 \mathrm{~ms}$ pulsar (Navarro et al. 1995) in a 2-day orbit with a helium white dwarf companion, at a distance of $\approx 3 \mathrm{kpc}$. Its spin-down properties imply $\dot{E}=2.4 \times 10^{35} \mathrm{erg} \mathrm{s}^{-1}$, which places it among the four most energetic MSPs known. PSR J0218+4232 was probably detected by EGRET, making it an appealing target for X-ray observatories. Detections in the $0.1-2.4 \mathrm{keV}$ band with the ROSAT HRI and PSPC instruments were reported by Verbunt et al. (1996) and Kuiper et al. (1998). A subsequent study by Mineo et al. (2000) resulted in measured pulsed emission in the 1-10 keV band in BeppoSAX observations. Follow up investigations have also been conducted with Chandra (Kuiper et al. 2002) and XMM-Newton (Webb et al. 2004), which confirm the previous findings of two moderately sharp pulses per period with a hard non-thermal spectrum.

PSR J0218+4232 is only just bright enough to correct the photon arrival times for the clock irregularities using the method of 22.2 . Although the pulse is not as sharp and the signal not is as strong per orbit as those for the our previous MSP examples, we were able to sucessfully recover the pulsed profile, but with reduced timing resolution.

\subsection{Observations and Results}

NuSTAR observed PSR J0218+4232 during Cycle 1 on 2015 October 28 (ObsID 30101030002; PI Ng) resulting in a $34.7 \mathrm{ks}$ effective exposure. The data for this pulsar were extracted and processed in the same way as the other two pulsars. For the pulsar timing analysis, we used the binary ephemeris from Abdo et al. (2010) to correct the photon arrival times for the pulsar orbit before applying the clock fixes.

We also retrieved archival Chandra data of PSR J0218+4232, consisting of a 72.1 ks HRC-S exposure from 2001 November 27 (ObsID 1853) first analyzed by Kuiper et al. (2002). Events were extracted from a circular aperture of radius $2^{\prime \prime}$ centered on the radio pulsar position. The photon arrival times were barycentered using the axbary tool in CIAO and folded with the same radio ephemeris used for the $N u S T A R$ event list.

The available archival XMM-Newton data PSR J0218+4232 is from a $37 \mathrm{ks}$ exposure obtained on 2002 February 11 (ObsID 0111100101) and is originally presented in Webb et al. (2004). The PN instrument was used in fast timing mode, while both MOS cameras were operated in full window mode. Roughly half of the observation is affected by strong background flaring, resulting in only $20 \mathrm{ks}$ of usable exposure time. To generate an event list suitable for timing and spectroscopy, we followed the same procedure as in 2.2

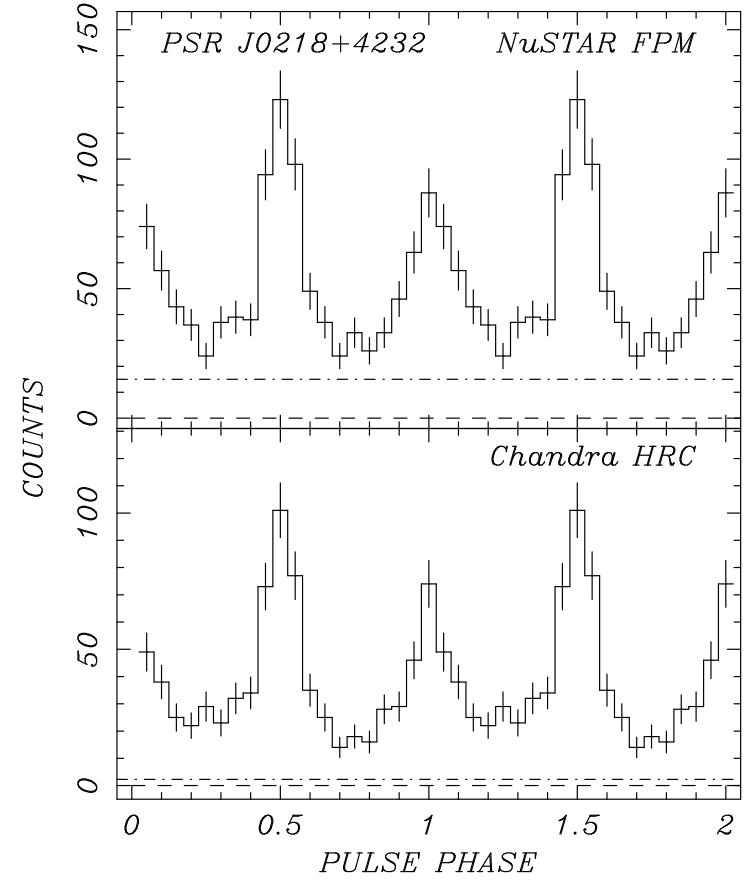

Fig. 8. - $2.32 \mathrm{~ms}$ pulse profiles of PSR J0218+4232 from (Top) NUSTAR FPMA+FPMB 3-79 keV photon arrival times corrected for spacecraft clock inaccuracies, and (Bottom) Chandra HRC-S data obtained in fast timing mode. The background is not subtracted. These profiles contain 20 phase bins and phase zero is arbitrarily aligned so that the pulsar peak $\mathrm{P} 1$ falls on $\phi=0.5$. Two cycles are shown for clarity.

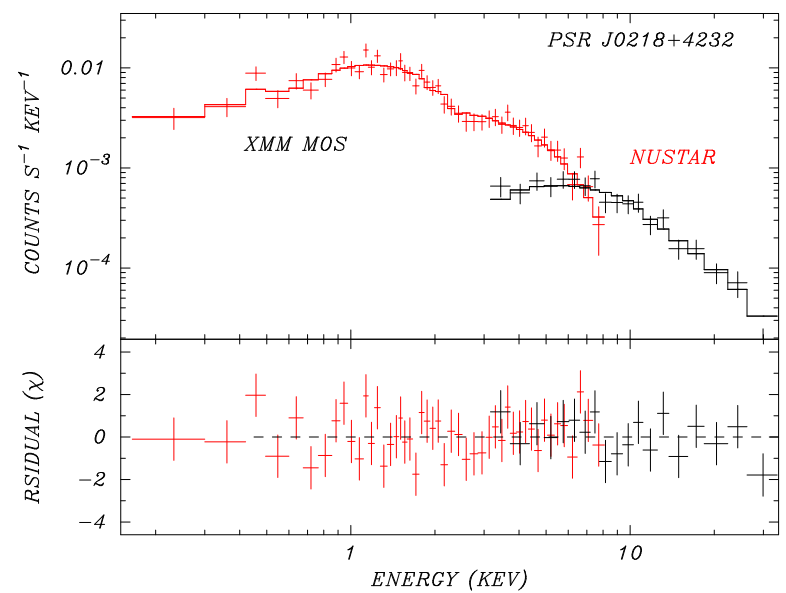

FIG. 9.- The NuSTAR FPMA+FPMB and XMM-Newton EPIC MOS1+MOS2 spectra of PSR J0218+4232. The data are fitted simultaneously to an absorbed power-law model with their normalizations left to vary independently. The upper panel present $N u S$ $T A R$ (red) and XMM-Newton (black) spectral histograms along with the best-fit model (solid lines) given in the text and Table . The lower panel shows the residuals from the best-fit model in units of sigma.

As shown in previous works Kuiper et al. 2002; Webb et al. 2004), the X-ray pulsations of PSR J0218+4232 are appreciably broader compared to those observed from PSRs B1821-24 and B1937+21. In addition, there is evidence for a bridge of emission connecting the two pulse peaks, with no obvious off-pulse interval. Compared to the other two MSPs, the pulsed signal for PSR J0218+4232 is not consistently detectable 
during a $N u S T A R$ orbit with the same quality, allowing the possibility of a $\sim 1 / 2$ cycle phase ambiguity for some $(\sim 1-2)$ measurements. Nevertheless, our approach for correcting the photon arrival times is able to recover the accurate pulse profile in $0.1 \mathrm{~ms}$ phase bins, as shown in Figure 8. Mineo et al. (2000) have presented evidence for the smaller pulse (P2) becoming stronger at higher energies. However, within the limits of the photon statistics, we see no indications for this in the NuSTAR profile above $\approx 10 \mathrm{keV}$.

For PSR J0218+4232 we again extracted a NuSTAR spectrum of the pulsed emission only, centered on the two pulse peaks $(3+4$ phase bins). For the lack a clear offpulse interval to represent the background, in this case we instead used the phase-averaged background spectrum, scaled to the on-pulse interval ( $7 / 20$ phase bins). We fit this NuSTAR spectrum simultaneously with the phaseaveraged XMM-Newton EPIC MOS spectra and, as expected, an absorbed power-law model results in a good fit with $\Gamma=1.10 \pm 0.09, N_{\mathrm{H}}=(5.2 \pm 3) \times 10^{20} \mathrm{~cm}^{-2}$, with $\chi_{\nu}^{2}=0.88$ for 63 DoF. By comparing the XMMNewton and NuSTAR best fit fluxes, we find that the bridge emission contributes $\lesssim 35 \%$ to the total in the $2-10 \mathrm{keV}$ band. The lower limit allows for possible imperfections in timing reconstruction which might add to the bridge emission.

\section{BROADBAND SPECTRAL ENERGY DISTRIBUTION}

Using the improved constraints on the hard portion of the X-ray spectra for the three MSPs, we can reexamine the spectral energy distribution (SED) of these objects ranging from soft $\mathrm{X}$-rays to $\mathrm{GeV}$ gamma-rays by combining our X-ray results with information in the high energy $\gamma$-ray range obtained with Fermi LAT.

Johnson et al. (2013) reported on the detection of $\gamma$ ray pulsations from PSR B1821-24 at a level of $5.4 \sigma$ in Fermi LAT data. The $\gamma$-ray pulsations are misaligned from the radio and $\mathrm{X}$-ray peaks as is common among MSPs. The $\gamma$-ray spectrum is well fit by an exponentially cutoff typical of pulsars, with power-law with $\Gamma=1.6 \pm$ 0.3 and cutoff energy $3.3 \pm 1.5 \mathrm{GeV}$.

For PSR B1937+21, an analysis of Fermi LAT data is presented by Guillemot et al. (2012). They find pulsed $\gamma$-ray emission that can be described by an exponentially cutoff power-law with $\Gamma=1.43 \pm 0.87$ and a fairly low cutoff energy $1.15 \pm 0.74 \mathrm{GeV}$.

PSR J0218+4232 is one of the first MSPs detected with Fermi LAT. Its $\gamma$-ray spectrum is much better characterized compared to the other two pulsars; it can be fit by an exponentially cutoff power-law with index $\Gamma=2.0 \pm 0.1$ and cutoff energy 4.6 $\pm 1.2 \mathrm{GeV}$ (Abdo et al. 2013).

The SED of the three MSPs spanning $0.1 \mathrm{keV}$ to $\sim 60$ $\mathrm{GeV}$ are shown in Figure 10. It is apparent that extrapolation of the power-law spectrum from the X-ray range to higher energies greatly over-predicts the flux in the highenergy $\gamma$-ray range observed by Ferm $i$ LAT. This is an indication that a break or smooth turnover must occur between the NuSTAR and Fermi data. The addition of the NuSTAR data, which shows no evidence for a deviation from a power-law, reveals that any turnover in the powerlaw spectrum occurs above $\sim 100 \mathrm{keV}$. Due to the absence of a telescope with sufficient sensitivity in the $100 \mathrm{keV}$ to $100 \mathrm{MeV}$ range, we cannot constrain the spectrum of PSR B1821-24 in this range. For PSR B1821-24,
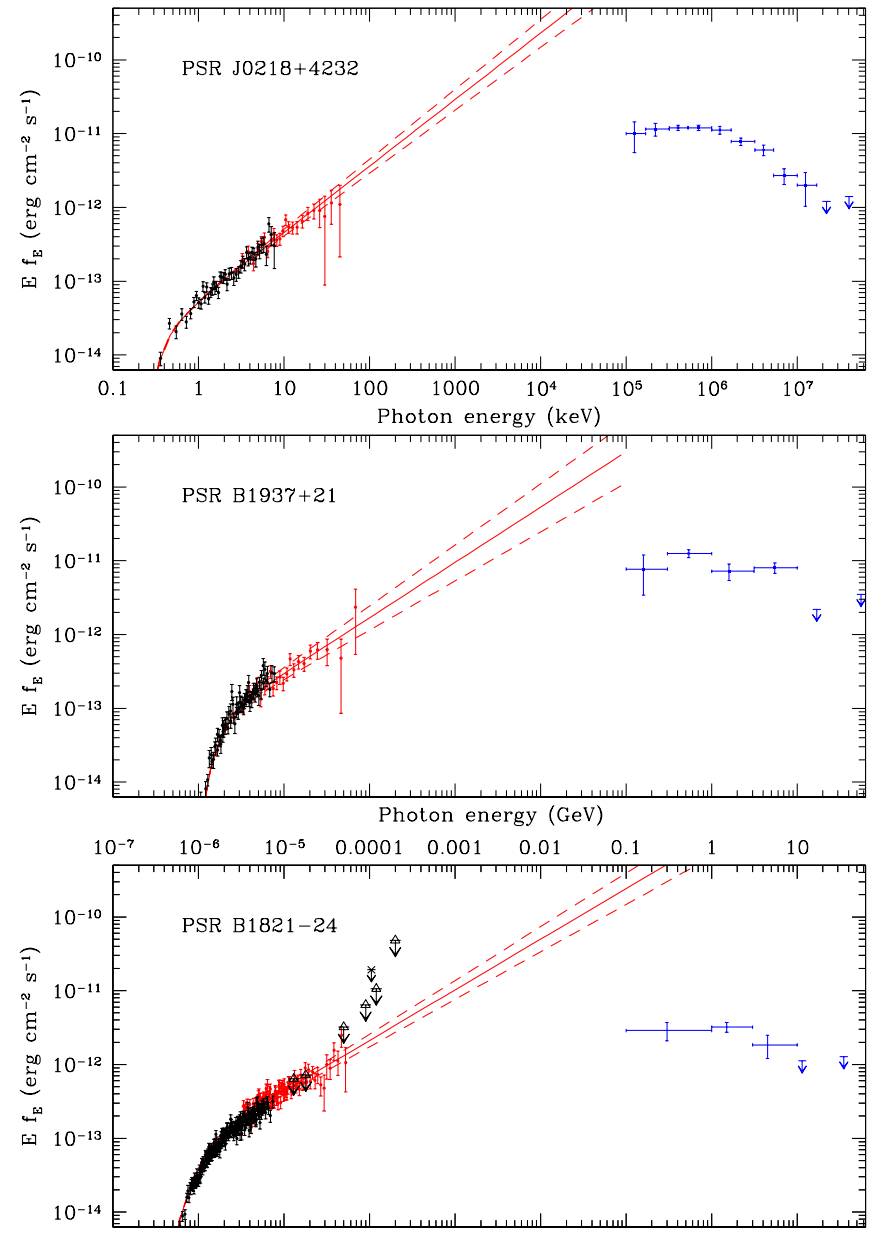

FIG. 10.- Top: The broad band spectral energy distribution of PSR B1821-24 spanning the soft X-ray to high-energy $\gamma$-ray range. The Chandra ACIS-S (black) and NuSTAR FPMA+FPMB (red) unfolded spectra of PSR B1821-24, fitted jointly with an absorbed power-law model are shown. The blue squares and upper limit arrows show the Fermi LAT data for photon energies $\geq 100 \mathrm{MeV}$ from [Johnson et al. (2013). The open triangles and cross show the HEXTE and OSSE upper limits from Kuiper et al. (2004). Middle: Same as top panel but for PSR B1937+21. The Fermi LAT spectrum is from Ng et al. (2014). Bottom: Same as top panel but for PSR J0218+4232. The Fermi LAT spectrum is from Abdo et al. (2013).

the presently available data for energies $\gtrsim 100 \mathrm{keV}$ from HEXTE, OSSE (from Kuiper et al. 2004) only provide upper limits on the flux that are not in conflict the extrapolation of the X-ray power-law.

The broad band spectral energy distributions of the three MSPs are reminiscent of the high energy emission from the Crab pulsar. In X-rays, the Crab pulsar also shows a purely non-thermal spectrum with $\Gamma=$ 1.6, which appears to start turning over smoothly at $\sim 100 \mathrm{keV}$ and is observed up to $\sim 300 \mathrm{MeV}$ (see, e.g., Bühler \& Blandford 2014, and references therein). Beyond that, an exponentially cut-off power-law begins to dominate the spectrum.

There exist a variety of theoretical models of pulsar electrodynamics that are used to interpret the observed high energy magnetospheric emission from pulsars. They differ principally in the assumed location 
TABLE 2

Summary OF SPECTROSCOPIC ANALYSIS FOR THREe MSPS

\begin{tabular}{|c|c|c|c|c|c|c|}
\hline Pulsar & $\left(10^{21} \mathrm{~cm}^{-2}\right)$ & $\Gamma$ & $\begin{array}{c}F_{X}^{1} \\
(0.3-8 \mathrm{keV})\end{array}$ & $\begin{array}{c}F_{X}^{1} \\
(2-10 \mathrm{keV})\end{array}$ & $\begin{array}{c}F_{X}{ }^{1} \\
(3-79 \mathrm{keV})\end{array}$ & $\chi^{2} /$ dof \\
\hline B1821-24 Chandra+NuSTAR & $4.01 \pm 0.04$ & $1.28 \pm 0.05$ & $3.19 \pm 0.09[\mathrm{C}]$ & $3.69 \pm 0.19[\mathrm{~N}]$ & $22.1 \pm 1.5[\mathrm{~N}]$ & $0.91 / 226$ \\
\hline B1937+21 Chandra+XMM-Newton $+N u S T A R$ & $17.8 \pm 2.7$ & $1.16 \pm 0.11$ & $3.01 \pm 0.18[\mathrm{X}]$ & $2.87 \pm 0.21[\mathrm{X}]$ & $18.2 \pm 2.8[\mathrm{~N}]$ & $0.91 / 83$ \\
\hline J0218+4232 XMM-Newton+NuSTAR & $0.52 \pm 0.3$ & $1.10 \pm 0.09$ & $3.97 \pm 0.26[\mathrm{X}]$ & $3.88 \pm 0.35[\mathrm{X}]$ & $19.8 \pm 2.3[\mathrm{~N}]$ & $0.88 / 63$ \\
\hline
\end{tabular}

${ }^{a}$ Unabsorbed flux in units of $10^{-13} \mathrm{erg}_{\mathrm{cm}^{-2}} \mathrm{~s}^{-1}$, for the [C] Chandra, [X] XMM-Newton, or [N] NuSTAR spectral component of the composite fit. Chandra spectra likely suffers from pile up.

of particle acceleration and attendant emission: polar cap (e.g., Daugherty \& Harding 1996), outer gap (e.g, Romani 1996), slot gap (Muslimov \& Harding 2004), or beyond the light cylinder in the current sheet (see, e.g., Cerutti et al. 2016). In the context of these models where the pulsed high-energy emission originates within the magnetosphere, this can be understood in terms of a spectrum transitioning from a synchrotron component at soft and hard X-ray energies to dominant curvature radiation in $\gamma$-rays or synchrotron emission from the current sheet. The improved constraints on the hard X-ray spectra of the three MSPs considered here provide additional information regarding the physics of the pulsar magnetosphere. Any viable model needs to account for both the pulse shapes and the spectral energy distribution.

\section{CONCLUSIONS}

We have presented detailed timing and spectroscopic analyses of the NuSTAR data of the energetic MSPs PSRs B1821-24, B1937+21, and J0218+4232. By making use of the sharpness of their pulses, bright enough to detect in individual orbits, we are able to phase align the pulse signal between orbit gaps. By this means we are able negate the significant drift in the $N u S T A R$ clock during these gaps, likely cause by solar illumination. This allowed a phase-resolved analysis of all three pulsars.

It is important to note the limitations of this approach. The applied clock corrections are only valid during the course of an observation since the clock drift by $\sim$ milliseconds occurs on timescales comparable to the $N u S T A R$ orbit (97 minutes). In addition, the clock correction method benefits from sharp pulsations; for fainter pulsars with broader modulations, the pulsations may not be detectable on intervals of order the telescope orbit (see, e.g., Guillot et al. 2016 for the case of PSR J0437-4715).

Spectra obtained with $N u S T A R$ allow us to measure all three pulsars to higher energies than possible with $R X T E$, in comparable exposures, highlighting the advantage of focusing optics, that results in the substantially lower background. At these higher energies we find the same power-law seen at lower energies with no evidence for a spectral break or turnover. Furthermore, the pulse profiles for all three pulsars are found to be essentially invariant with energy, from the soft to hard X-ray bands.
When combined with the GeV data from Fermi LAT, we have obtained the best yet measurements of the broadband spectral shape and high-energy pulsed emission of each MSP. We find that in all cases the power-law spectrum needs to turn over somewhere between $100 \mathrm{keV}$ and $100 \mathrm{MeV}$ to be consistent with the high energy $\gamma$-ray spectrum. This can be understood in terms of a spectrum transitioning from a dominant synchrotron component in $\mathrm{X}$-rays to curvature radiation or current sheet emission in $\gamma$-rays, very similar to the Crab pulsar.

We thank S. Ransom for supplying the radio ephemeris of PSR B1821-24. This work was supported under NuSTAR Cycle 1 Guest Observer Program grant NNX15AV29G awarded through Columbia University. E.V.G. thanks Josep Maria Paredes for hosting his sabbatical at the University of Barcelona Institut de Ciències del Cosmos (ICCUB) and acknowledges support through the "Programa Estatal de Foment de la Investigaciò Científica i Tècnica d'Excellència, Convocatòria 2014, Unitats d'Excellència Maria de Maeztu". The NuSTAR mission is a project led by the California Institute of Technology, managed by the Jet Propulsion Laboratory, and funded by the National Aeronautics and Space Administration. We thank the $N u S T A R$ Operations, Software and Calibration teams for support with the execution and analysis of these observations. This research made uses of the NuSTAR Data Analysis Software (NuSTARDAS) jointly developed by the ASI Science Data Center (ASDC, Italy) and the California Institute of Technology (USA). The scientific results reported in this article are based in part on data obtained from the Chandra Data Archive. A portion of the results presented are based on observations obtained with XMM-Newton, an ESA science mission with instruments and contributions directly funded by ESA Member States and NASA. This research has made use of data and software provided by the High Energy Astrophysics Science Archive Research Center (HEASARC), which is a service of the Astrophysics Science Division at NASA/GSFC and the High Energy Astrophysics Division of the Smithsonian Astrophysical Observatory. We also acknowledge extensive use of the arXiv and the NASA Astrophysics Data Service (ADS).

Facilities: NuSTAR, Chandra, XMM-Newton

\section{REFERENCES}

Abdo, A. A., Ackermann, M., Ajello, M. 2010, ApJS, 187, 460 Abdo, A. A., Ajello, M., Allafort, A., et al. 2013, ApJS, 208, 17 Arnaud, K. A. 1996, ASPC, 101, 17
Backer, D. C., Kulkarni, S. R., Heiles, C., Davis, M. M., Goss, W. M. 1982, Nature, 300, 615 
Bahramian, A., Heinke, C. O., Tudor, V., et al. 2017, MNRAS, 467, 2199

Becker, W., Swartz, D. A., Pavlov, G. G., Elsner, R. F., Grindlay, J. E., Mignani, R., Tennant, A. F., Backer, D., Pulone, L., Testa V., \& Weisskopf, M. C. 2003, ApJ, 594, 798

Bégin, S., Thesis submitted to the Faculty of Physics, University of British Columbia, 2006

Bogdanov, S., Esposito, P., Crawford, F., III, Possenti, A. McLaughlin, M. A., Freire, P. 2014, ApJ, 781, 6

Bogdanov, S., Grindlay, J. E., \& van den Berg, M. 2005, ApJ, 630, 1029

Bogdanov, S., Grindlay, J. E., Heinke, C. O., Camilo, F., Freire P. C. C., \& Becker, W. 2006, ApJ, 646, 1104

Bogdanov, S., van den Berg, M., Servillat, M., Heinke, C. O. Grindlay, J. E., Stairs, I. H., Ransom, S. M., Freire, P. C. C., Bégin, S., Becker, W. 2011, ApJ, 730, 81

Bühler, R., \& Blandford,R. 2014, RPPh, 77, 6901

Cerutti, B., Philippov, A. A., Spitkovsky, A. 2016, MNRAS, 457, 2401

Daugherty, J. K., Harding, A. K. 1996, ApJ, 458, 278

Desvignes, G., Caballero, R. N., Lentati, L., et al. 2016, MNRAS, 458,3341

Forestell, L. M., Heinke, C. O., Cohn, H. N., Lugger, P. M., Sivakoff, G. R., Bogdanov, S., Cool, A. M., Anderson, J. 2014, MNRAS, 441,757

Guillemot, L., Johnson, T. J., Venter, C., et al. 2012, ApJ, 744, 33

Guillot, S., Kaspi, V. M., Archibald, R. F., et al. 2016, MNRAS, 463,2612

Muslimov, A. G. 2002, ApJ, 568, 862

Muslimov, A. G. 2005, ApJ, 622, 531

Harrison, F. A., Craig, W. W., Christensen, F. E. et al. 2013, ApJ, 770,103

Jahoda, K., Swank, J. H., Giles, A. B., Stark, M. J., Strohmayer, T., Zhang, W., Morgan, E. H. 1996, Proc. SPIE, 2808, 59

Johnson, T. J., Guillemot, L., Kerr, M., et al. 2013, ApJ, 778, 106

Kuiper, L., Hermsen, W., Stappers, B. 2004, AdSpR, 33, 507

Kuiper, L., Hermsen, W., Verbunt, F., Belloni, T. 1998, A\&A, 336, 545

Kuiper, L., Hermsen, W., Verbunt, F., Ord, S., Stairs, I., Lyne, A. 2002, ApJ, 577, 917

Linares, M., Bahramian, A., Heinke, C., Wijnands, R., Patruno, A., Altamirano, D., Homan, J., Bogdanov, S., Pooley, D. 2014 MNRAS, 438, 251

Lyne, A. G., Brinklow, A., Middleditch, J., Kulkarni, S. R., Backer, D. C. 1987 , Nature, 328, 399
Madsen, K. K., Harrison, F. A., Markwardt, C. B., et al. 2015, ApJS, 220, 8

Mineo, T., Cusumano, G., Kuiper, L., Hermsen, W., Massaro, E. Becker, W., Nicastro, L., Sacco, B., Verbunt, F., Lyne, A. G., Stairs, I. H., Shibata, S. 2000, A\&A, 355, 1053

Mori, K., Gotthelf, E. V., Dufour, F., et al. 2014, ApJ, 793, 88

Muslimov, A. G., Harding, A. K. 2004, ApJ, 606, 1143

Navarro, J., de Bruyn, A. G., Frail, D. A., Kulkarni, S. R., Lyne, A. G. 1995, ApJ, 455, L55

Ng, C.-Y., Takata, J., Leung, G. C. K., Cheng, K. S., Philippopoulos, P. 2014, ApJ, 787, 167

Nicastro, L., Cusumano, G., Löhmer, O., Kramer, M., Kuiper, L., Hermsen, W., Mineo, T., Becker, W. 2004, A\&A, 413, 1065

Pan, Z., Hobbs, G., Li, D., Ridolfi, A., Wang, P., Freire, P. 2016 MNRAS, 459, L26

Papitto, A., Ferrigno, C., Bozzo, E., et al. 2013, Nature, 501, 517

Ray, P. S., Wolff, M. T., Demorest, P., Cognard, I., Backer, D. C., Wood, K. S. 2008, AIPC, 983, 157

Romani, R. W. 1996, ApJ, 470, 469

Rots, A. H., Jahoda, L., Macomb, D. J., et al. 1998, ApJ, 501, 749

Rutledge, R. E. Fox, D. W., Kulkarni, S. R., Jacoby, B. A., Cognard, I., Backer, D. C., \& Murray, S. S. 2004, ApJ, 613, 522

Saito, Y., Kawai, N., Kamae, T., Shibata, S., Dotani, T., Kulkarni, S. R. 1997, ApJ, 477, L37

Servillat, M., Heinke, C.'O., Ho, W. C. G., Grindlay, J. E., Hong, J., van den Berg, M., Bogdanov, S. 2012, MNRAS, 423, 1556

Takahashi, M., Shibata, S., Torii, K., Saito, Y., Kawai, N., Hirayama, M., Dotani, T., Gunji, S., Sakurai, H., Stairs, I. H., Manchester, R. N. 2001, ApJ, 554, 316

Ubertini, P., Bazzano, A., Cocchi, M., Natalucci, L., Heise, J., Muller, J.' M., in't Zand, J. J. M. 1999, ApJ, 514, L27

Venter, C., Harding, A. K. \& Guillemot, L. 2010, ApJ, 707, 800

Verner, D. A., Ferland, G. J., Korista, K. T., Yakovlev, D. G. 1996 ApJ, 465, 487

Verbunt, F., Kuiper, L., Belloni, T., Johnston, H .M., de Bruyn, A. G., Hermsen, W., van der Klis, M. 1996, A\&A, 311, L9

Webb, N. A. Olive, J. -F., \& Barret, D. 2004, A\&A, 417, 181

Wilms, J., Allen, A., \& McCray, R. 2000, ApJ, 542, 914

Zavlin, V. E. 2006, ApJ, 638, 951

Zavlin, V. E. 2007, Ap\&SS, 308, 297 